\title{
Coordination of hypothalamic and pituitary T3 production regulates TSH expression
}

Tatiana L. Fonseca, ${ }^{1}$ Mayrin Correa-Medina, ${ }^{1}$ Maira P.O. Campos, ${ }^{1}$ Gabor Wittmann, ${ }^{2}$ Joao P. Werneck-de-Castro, ${ }^{1,3}$ Rafael Arrojo e Drigo, ${ }^{1}$ Magda Mora-Garzon, ${ }^{1}$ Cintia Bagne Ueta, ${ }^{1}$ Alejandro Caicedo, ${ }^{1}$ Csaba Fekete, ${ }^{2,4}$ Balazs Gereben, ${ }^{4}$ Ronald M. Lechan, ${ }^{2}$ and Antonio C. Bianco ${ }^{1}$

\author{
${ }^{1}$ Division of Endocrinology, Diabetes and Metabolism, Miller School of Medicine, University of Miami, Miami, Florida, USA. \\ 2Department of Medicine, Division of Endocrinology, Diabetes and Metabolism, Tufts Medical Center, Boston, Massachusetts, USA \\ 3Institute of Biophysics Carlos Chagas Filho and School of Physical Education and Sports, Federal University of Rio de Janeiro, \\ Rio de Janeiro, Brazil. ${ }^{4}$ Department of Endocrine Neurobiology, Institute of Experimental Medicine, \\ Hungarian Academy of Sciences, Budapest, Hungary.
}

\begin{abstract}
Type II deiodinase (D2) activates thyroid hormone by converting thyroxine (T4) to 3,5,3'-triiodothyronine (T3). This allows plasma T4 to signal a negative feedback loop that inhibits production of thyrotropin-releasing hormone (TRH) in the mediobasal hypothalamus (MBH) and thyroid-stimulating hormone (TSH) in the pituitary. To determine the relative contributions of these $\mathrm{D} 2$ pathways in the feedback loop, we developed 2 mouse strains with pituitary- and astrocyte-specific $D 2$ knockdown (pit-D2 KO and astro- $D 2 \mathrm{KO}$ mice, respectively). The pit-D2 KO mice had normal serum T3 and were systemically euthyroid, but exhibited an approximately 3-fold elevation in serum TSH levels and a $40 \%$ reduction in biological activity. This was the result of elevated serum T4 that increased D2-mediated T3 production in the MBH, thus decreasing Trb mRNA. That tanycytes, not astrocytes, are the cells within the MBH that mediate T4-to-T3 conversion was defined by studies using the astro-D2 KO mice. Despite near-complete loss of brain D2, tanycyte D2 was preserved in astro-D2 KO mice at levels that were sufficient to maintain both the T4-dependent negative feedback loop and thyroid economy. Taken together, these data demonstrated that the hypothalamic-thyroid axis is wired to maintain normal plasma T3 levels, which is achieved through coordination of T4-to-T3 conversion between thyrotrophs and tanycytes.
\end{abstract}

\section{Introduction}

The negative feedback loop between circulating thyroid hormone and the neuroendocrine system (hypothalamus and pituitary gland) is the fundamental mechanism preserving thyroid hormone homeostasis. During iodine deficiency, there is decreased synthesis of thyroid hormone that leads to a reduction in plasma thyroxine (T4), rapidly activating secretion of hypothalamic thyrotropin-releasing hormone (TRH) and pituitary thyroid-stimulating hormone (TSH), which stimulates the thyroid to grow and normalize thyroid hormone synthesis and secretion (1). As with T4, a reduction in plasma 3,5,3'-triiodothyronine (T3), the biologically active thyroid hormone, also triggers the feedback mechanism and activates TRH and TSH secretion. This has been well documented in patients acutely given large amounts of propylthyouracyl (PTU), which inhibits peripheral synthesis of T3 (2).

Given that the TSHB and TRH genes are negatively regulated by $\mathrm{T} 3$ (and not T4), it is crucial that T4 be converted to T3 in order to activate the negative feedback mechanism. The presence of type 2 deiodinase (D2), which converts T4 to T3 in the brain, is thought to be critical for this mechanism (3). D2 is coexpressed with TSH in pituitary cells (4) and is also expressed in astrocytes in thyroid hormone-sensitive regions of the brain, such as the cerebral cortex and hippocampus $(5,6)$, but its presence has also been documented in astrocytes in the mediobasal hypothalamus $(\mathrm{MBH})(7)$. However, the highest expression of D2 in the MBH is in tanycytes, specialized ependymal cells lining the floor and infralateral wall of the third ventricle. In fact, tanycytes and TRH-expressing neurons in

Conflict of interest: The authors have declared that no conflict of interest exists. Citation for this article: J Clin Invest. 2013;123(4):1492-1500. doi:10.1172/JCI61231. the paraventricular nucleus $(\mathrm{PVN})$ are intermingled in the external zone of the median eminence $(8,9)$, providing the physical proximity for D2-mediated feedback signaling. Thus, hypothalamic D2 (astrocytic and tanycytic) has been proposed to have an important role in feedback regulation of thyroid hormone on hypophysiotropic TRH (10), but the relative importance of D2 expression in astrocytes versus tanycytes is not known.

Global inactivation of the D2 gene (also known as Dio2) in mice leads to elevated serum TSH and T4, but serum T3 remains normal, a phenotype compatible with an impaired transduction mechanism in T4-mediated TSH suppression (11). A very similar phenotype was also observed in mice treated with amiodarone, a noncompetitive inhibitor of D2 (12). However, neither the global D2 KO mouse nor the amiodarone-treated mouse models allow for a mechanistic understanding of the relative roles played by pituitary versus hypothalamic D2 in the thyroid hormone-mediated feedback mechanism. In addition, an unexpected finding was that both of these animal models exhibit normal PVN TRH expression, calling into question the role played by hypothalamic D2 in the T4-mediated feedback mechanism.

To address these questions, we developed the floxed D2 $\left(D 2^{f t}\right)$ mouse and used it here to generate 2 mouse strains: one exhibiting near-null $D 2$ activity in the pituitary gland (referred to herein as pitD2 KO mice), and one lacking D2 activity selectively in astrocytes (astro-D2 KO mice). We showed that the disruption in the T4-mediated mechanism of the pit- $D 2 \mathrm{KO}$ mouse was bypassed by elevated serum T4 and reduced PVN TRH expression, defining a critical role for the MBH D2 pathway in the feedback mechanism. The phenotypic characterization of astro- $D 2 \mathrm{KO}$ mice indicated that tanycytes, rather than astrocytes, are the cell type within the $\mathrm{MBH}$, where this critical D2-mediated T4-to-T3 conversion takes place. 


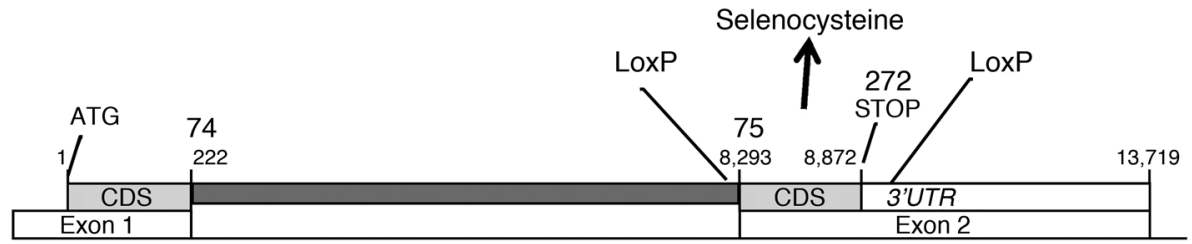

\section{Figure 1}

D2 gene. The 2 loxP sites flanking the selenocysteine insertion site, base numbers, and amino acid positions are indicated. ATG, methionine; CDS, coding sequence.

\section{Results}

Pituitary-specific D2 inactivation disrupts the bypothalamic-pituitarythyroid axis (HPT). To determine the importance of pituitary versus hypothalamic D2 in HPT regulation, we used p-flox technology to allow for tissue-specific inactivation of the $D 2$ gene based on selective expression of Cre recombinase (see Methods and Figure 1). We used mice expressing Cre recombinase under the pituitary-specific glycoprotein hormone $\alpha$-subunit promoter (CGA-cre mice), which led to the generation of pit-D2 KO mice (CGA-cre/D2fl), a strain in which D2 activity was markedly reduced in the pituitary while remaining unaffected in the other D2-expressing tissues (i.e., cerebral cortex, hypothalamus, hippocampus, brown adipose tissue (BAT), and placenta; Figure 2, A-E, and Supplemental Figure 1M; supplemental material available online with this article; doi:10.1172/JCI61231DS1). D3 activity was unaffected throughout the brain (Supplemental Figure 1, A-C).

The pit-D2 KO mouse exhibited about 2.8-fold higher serum TSH levels and approximately $40 \%$ higher serum T4 levels com- pared with CGA-cre littermate controls, whereas serum T3 concentrations were not different (Figure 2, F, G, and I). No differences in ${ }^{125} \mathrm{I}-\mathrm{T} 4$ charcoal uptake were detected between pit-D2 $\mathrm{KO}$ and littermate control animals (Figure $2 \mathrm{H}$ ), which indicates that the higher serum T4 was associated with elevated free T4 levels. However, that the pit- $D 2 \mathrm{KO}$ mouse is systemically euthyroid was confirmed by studying tissue markers of thyroid hormone action. Total BW and individual organ weights in 2-month-old pit-D2 KO animals were similar to those of CGA-cre controls (Table 1). In addition, there were no differences in total skeleton bone mineral density (BMD), lean body mass (LBM), or oxygen consumption $\left(\mathrm{VO}_{2}\right)$ (Tables 2 and 3 ), all sensitive markers of thyroid hormone action $(13,14)$. The very sensitive genetic marker of thyroid hormone signaling, liver D1 activity (15), was unaffected in pit-D2 KO mice (Figure 2J). The only noticeable difference was the approximately $20 \%$ lower body fat mass in pit-D2 KO animals (Table 2). Given that CGA is also expressed in gonadotrophs, we confirmed that the gonadal axis was pre-
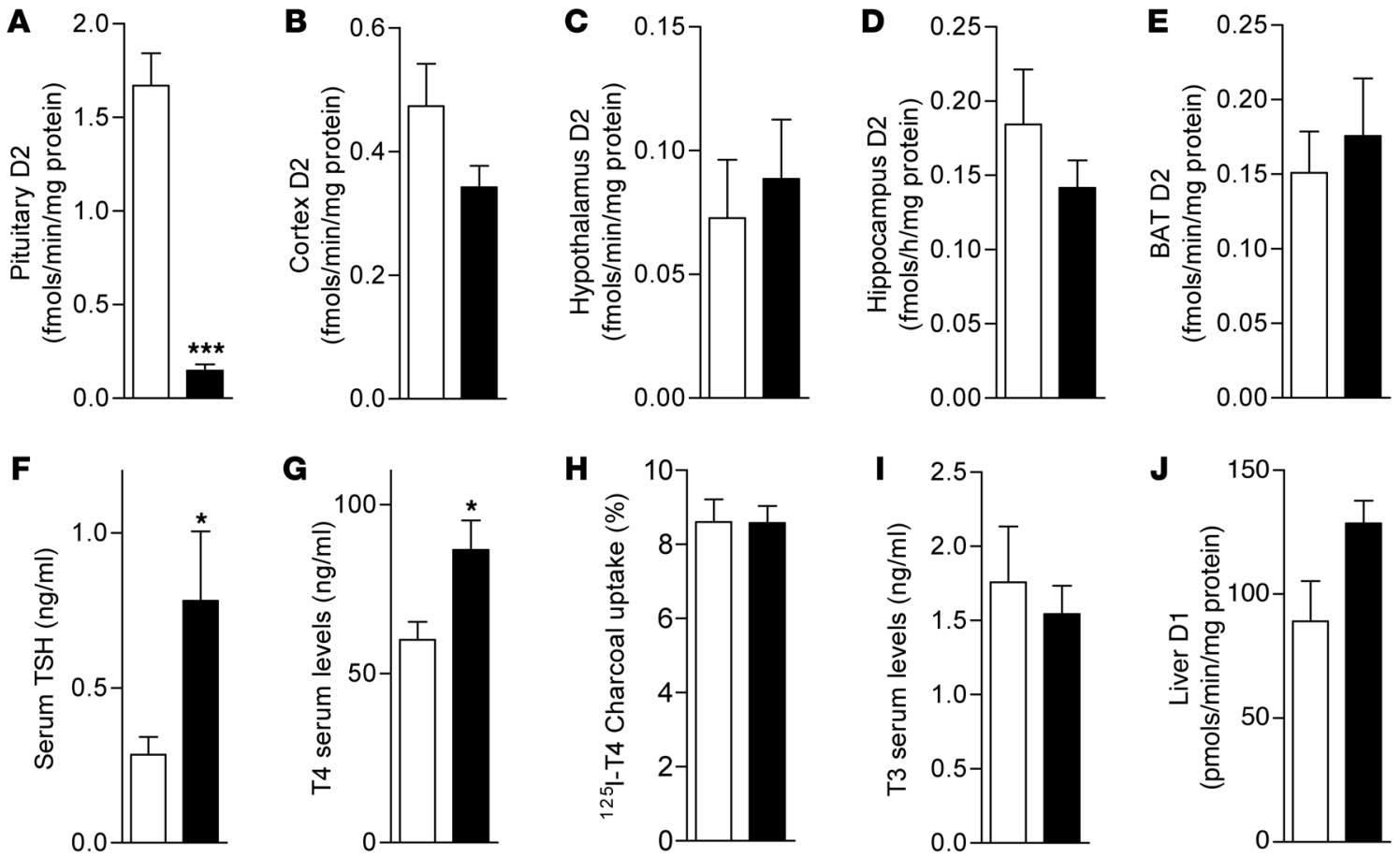

Figure 2

Deiodinase activity and thyroid function tests in pit-D2 KO mice versus CGA-cre controls. (A-E) D2 activity in sonicates of pituitary, cortex, hypothalamus, hippocampus, and BAT $(n=3-5)$. (F and G) Serum TSH and T4 levels $(n=9-10)$. (H) ${ }^{125}$-T4 charcoal uptake in the serum $(n=9)$. (I) Serum T3 levels $(n=9-10)$. (J) Liver D1 activity $(n=3-5)$. ${ }^{*} P<0.05,{ }^{* * *} P<0.001$ vs. control. 
Table 1

BW and organ weights

\begin{tabular}{lccccc} 
Group & BW $(\mathbf{g})$ & \multicolumn{3}{c}{ Organ weights (mg/mg BW) } \\
& & Brain & Epididymal fat & Liver & Muscle \\
& & $14 \pm 1.5$ & $27 \pm 10.2$ & $44 \pm 6.7$ & $5.3 \pm 0.7$ \\
CGA-cre control & $27 \pm 3.8$ & $12 \pm 0.58$ & $24 \pm 4.2$ & $49 \pm 3.1$ & $5.6 \pm 0.3$ \\
Pit-D2 KO & $29 \pm 2.0$ & $15 \pm 1.8$ & $13 \pm 4.4$ & $50 \pm 6.3$ & $5.4 \pm 0.4$ \\
GFAP-cre control & $28 \pm 2.0$ & $15 \pm 2.2$ & $20 \pm 1.4$ & $45 \pm 3.6$ & $5.8 \pm 0.3$ \\
Astro-D2 KO & $29 \pm 3.4$ & & &
\end{tabular}

Values are mean \pm SD of 5 animals.

served in pit-D2 $\mathrm{KO}$ mice, as shown by their normal serum levels of follicle-stimulating hormone (FSH) and luteinizing hormone $(\mathrm{LH})$ as well as normal gonadal weight compared with controls (Supplemental 1, G-J).

We next hypothesized that reducing D2 expression in thyrotrophs would render these cells less sensitive to T4-mediated signaling, as has been proposed for the global D2 KO mouse (11). This was tested by assessing acute TSH responsiveness to T4 or T3. In control animals, administration of T4 elevated serum T4 approximately 7 -fold and serum T3 approximately 3 -fold, but reduced serum TSH by about 10 -fold, to near-suppression levels $(0.08 \pm 0.002 \mathrm{ng} / \mathrm{ml})$ (Figure $3, \mathrm{~A}-\mathrm{C})$. The drop in serum TSH occurred as a result of T4-to-T3 conversion in the thyrotroph and elevated serum T3. In contrast, administration of the same dose of $\mathrm{T} 4$ in pit- $D 2 \mathrm{KO}$ animals only reduced serum TSH about 4-fold $(0.5 \pm 0.11 \mathrm{ng} / \mathrm{ml})$, despite similar elevations in serum T3 and serum T4 compared with control animals (Figure 3, A-C). This can only be attributed to impaired thyrotroph T4-to-T3 conversion, given the identical T3-induced TSH suppression in both groups (Figure 3C).

Notably, there were no differences between groups in 30-minute TRH-stimulated TSH secretion: in all animals, serum TSH increased to similar levels, although the calculated fold response to TRH was greater in the control animals $(\sim 7$ - versus $\sim 2$-fold), given the higher baseline serum TSH in pit-D2 KO mice (Figure 3D). Furthermore, serum levels of T4 and T3 remained unaffected in these TRH-treated animals (Supplemental Figure 1, K and L).

To test D2-mediated T4 signaling in pit-D2 $\mathrm{KO} \mathrm{MBH}$, we studied PVN Trh mRNA levels in both mouse groups by in situ hybridization and found an approximate $50 \%$ reduction in pit- $D 2 \mathrm{KO}$ mice (Figure 3, E and F). However, Trh mRNA levels remained unaffected in the lateral hypothalamus of pit-D2 KO mice (Figure $3 \mathrm{G}$ ). Given that MBH D2 remained unaffected in pit-D2 KO animals (Figure 2C), the elevated serum T4 and consequent increase in MBH D2-generated T3 explains the decreased TRH expression. This notion was supported by the finding that the difference in PVN Trh mRNA was minimized in control versus pit- $D 2 \mathrm{KO}$ animals that were made systemically hypothyroid (105 \pm 13 vs. $76 \pm$ $20 \mathrm{AU} ; P>0.05)$. Notably, the expression of key enzymes involved in processing the TRH peptide remained unaffected in the pit- $D 2$ $\mathrm{KO}$ hypothalamus (Figure 3, H and I), which indicates that the decreased Trh mRNA is not neutralized by posttranslational mechanisms, thereby decreasing TRH peptide production. This finding illustrates a marked difference between the pit-D2 $\mathrm{KO}$ and global D2 KO mouse models, defining a major role for MBH D2 in the T4-mediated TRH feedback mechanism.
Reduced PVN TRH expression is expected to decrease hypothalamic stimulation of the thyrotroph; indeed, Tsha mRNA levels were reduced in pit-D2 $\mathrm{KO}$ mice (Figure $3 \mathrm{~J}$ ). However, pituitary $T s h b$ mRNA levels were not affected in this mouse model (Figure $3 \mathrm{~K}$ ), presumably because the negative effects of T3 on TSH $\beta$ are greater than those on $\operatorname{TSH} \alpha(16,17)$. In other words, whereas $T s h b$ was less stimulated by the hypothalamus, it was also less repressed by T3, due to inactivation of the local D2 pathway.

How could an elevation in serum TSH be reconciled with normal pituitary $T s h b$ mRNA levels? We hypothesized that the reduction in pit-D2 KO PVN TRH expression prolonged TSH half-life and decreased its biological activity by altering the TSH glycosylation pattern (18). To test this hypothesis, we used a previously characterized in vitro assay to estimate pit- $D 2 \mathrm{KO}$ TSH biological activity. Inducible cAMP production in $\mathrm{CHO}$ cells stably expressing TSH receptors approximately doubled with pit-D2 KO sera and decayed linearly with sera dilution (Figure 4 , A and B). However, relative to the higher gravimetric amounts of TSH used in the assay (Figure 4C), pit-D2 KO TSH exhibited about $40 \%$ lower biological activity (Figure 4D). This is supported by our observations that thyroid structure, 2-hour ${ }^{125}$ I uptake, and mRNA levels of sodium/iodide symporter (NIS) and thyroglobulin (Tg) were not different from those in control animals (Figure 4, E-I), despite the much higher serum TSH (Figure 2F). Notably, the pit-D2 KO thyroid gland was slightly enlarged, but the elevation in serum $\mathrm{T} 4$ and T3 observed 2 hours after bovine TSH administration was similar in both mouse groups (Figure 4, J and K).

Astrocyte-specific D2 inactivation does not affect HPT. The $\mathrm{MBH}$ contains high D2 activity levels, expressed in both astrocytes (7) and tanycytes $(5,6)$. To elucidate the contribution of astrocyte versus tanycyte D2 in the HPT, we created a second mouse strain expressing Cre recombinase driven by the glial fibrillary acidic protein (GFAP) promoter (GFAP-cre mice), a well-known marker for matured astrocytes that is rarely expressed in tanycytes (Figure $5 \mathrm{~A})$. Indeed, in the resulting astro- $D 2 \mathrm{KO}$ mice (GFAP-cre/ $D 2^{f l}$; see Methods), D2 mRNA levels in tanycytes were not altered compared with GFAP-cre littermate controls (Figure 5, B and C). However, astro- $D 2 \mathrm{KO}$ mice exhibited a marked decrease in brain D2 activity in areas where D2 is expressed in glial cells (such as the cerebral cortex, hippocampus, and cerebellum), but not in the hypothalamus or pituitary gland, where D2 activity remained unaffected (Figure 6, A-E). In addition, D2 activity was also preserved outside the central nervous system (e.g., in BAT; Figure 6F). As with the pit-D2 KO mouse, D3 activity was not affected throughout the astro-D2 KO brain (Supplemental Figure 1, D-F).

\section{Table 2}

Body composition

\begin{tabular}{lcrc} 
Group & BMD $\left(\mathbf{m g} / \mathbf{c m}^{2}\right)$ & LBM $(\mathbf{g})$ & Fat mass $(\mathbf{g})$ \\
CGA-cre control & $51.8 \pm 3.6$ & $18.6 \pm 2.4$ & $6.3 \pm 1.3$ \\
Pit-D2 KO & $51.5 \pm 2.4$ & $19.0 \pm 1.3$ & $5.0 \pm 0.7^{\mathrm{A}}$ \\
GFAP-cre control & $55.7 \pm 2.1$ & $22.4 \pm 1.8$ & $7.2 \pm 1.4$ \\
Astro-D2 K0 & $52.8 \pm 1.4^{\mathrm{A}}$ & $21.5 \pm 1.8$ & $6.2 \pm 1.3$ \\
\hline
\end{tabular}

Values are mean \pm SD of $5-7$ animals. ${ }^{A} P<0.05$ vs. respective control. 
Table 3

Indirect calorimetric analysis

\begin{tabular}{lcc} 
& \multicolumn{2}{c}{$\mathbf{V O}_{2}(\mathbf{m l} / \mathbf{k g} / \mathbf{h})$} \\
Group & Light & Dark \\
CGA-cre control & $7.3 \pm 1.1$ & $8.8 \pm 1.6$ \\
Pit-D2 K0 & $8.4 \pm 1.4$ & $9.8 \pm 1.6$ \\
GFAP-cre control & $6.7 \pm 1.0$ & $7.6 \pm 0.9$ \\
Astro-D2 K0 & $6.7 \pm 1.7$ & $8.4 \pm 2.1$ \\
\hline
\end{tabular}

Values are mean \pm SD of 6 animals. All entries are AU from AUC for individual animals.

We found that astro-D2 KO mice had normal HPT and thyroid economy, as evidenced by their normal serum levels of TSH, T4, and T3; thyroid morphology; and PVN Trb mRNA expression (Figure 6, G-L). That the animals were systemically euthyroid was confirmed by analysis of multiple tissue markers of thyroid hormone action in 2-month-old animals, including BW, organ weights, $\mathrm{LBM}$, fat mass, and $\mathrm{VO}_{2}$ (Tables 1-3). Finally, liver D1 activity was unaffected in astro- $D 2 \mathrm{KO}$ mice (Figure $6 \mathrm{M}$ ). In addition, we tested the relative sensitivity of TSH secretion to T4 or T3 and found that administration of either hormone suppressed serum TSH (Figure $6 \mathrm{~N}$ ), indicative of preserved HPT sensitivity to T4 in astro-D2 KO mice. As with pit-D2 $\mathrm{KO}$ animals, in both experiments, there were no differences between the serum levels of T4 and T3 achieved after administration of T4 or T3 (data not shown). An unexpected finding was a lower total body BMD in astro- $D 2 \mathrm{KO}$ versus GFAPcre control mice (Table 2), which suggests that brain D2 could be involved in bone metabolism or that $\mathrm{D} 2$ expression in certain GFAP-positive bone cells could play a previously unappreciated role in bone mass.

\section{Discussion}

The development and phenotypic characterization of 2 strains of tissue-specific D2 KO mice revealed a novel aspect of HPT regulation, namely, that a decrease in D2-generated T3 in the thyrotrophs can be compensated for by changes in hypophysiotropic Trh gene expression, presumably via an increase in D2-generated $\mathrm{T} 3$ in the $\mathrm{MBH}$. Decreasing TRH expression in the pit-D2 KO mouse nullified any effect of selective pituitary D2 deficiency on Tshb gene expression, which remained unaffected (Figure 3K). However, the reduction in Trh expression (Figure 3, E and F) is likely to alter the pattern of TSH glycosylation, explaining the observed decrease in TSH biological activity (Figure 4, A-D). $\mathrm{MBH}$ D2 expression is present in astrocytes and in tanycytes (5-7). Thus, our observation that thyroid economy was preserved in the astro-D2 KO mouse (Figure 6, G-I) indicates that tanycytic, and not astrocytic, D2 is required to transduce the
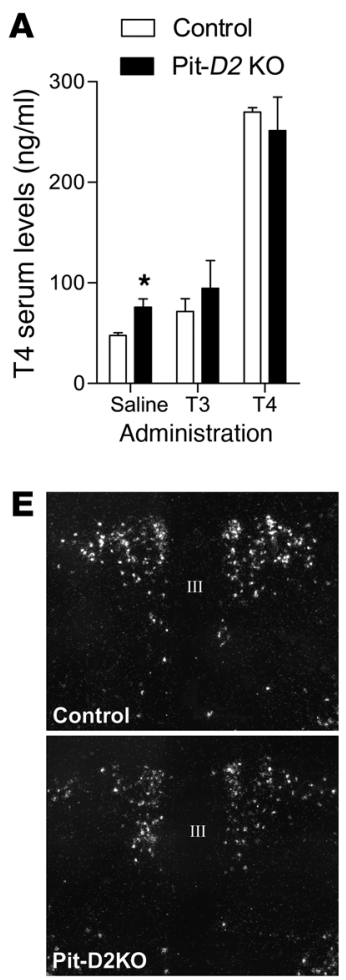

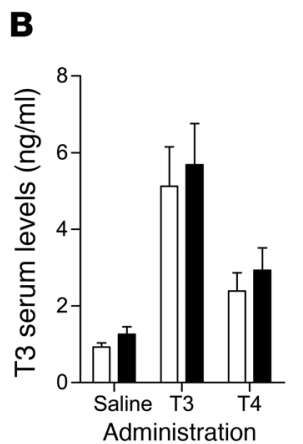

C

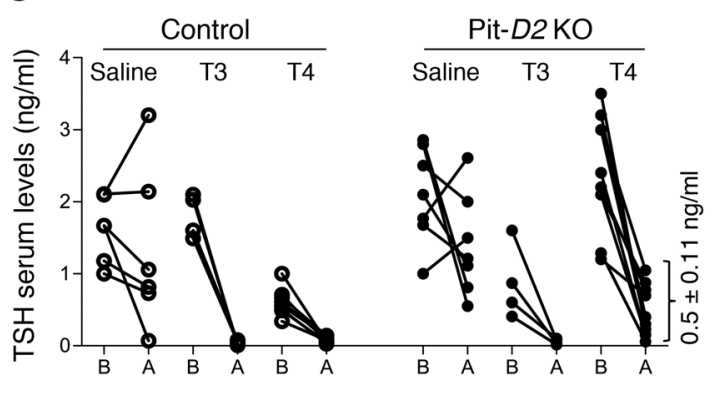

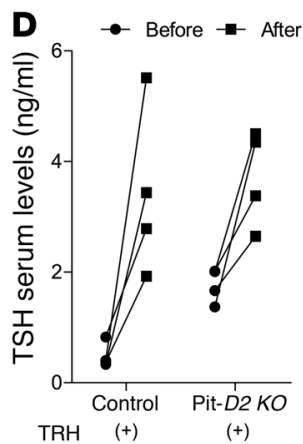

$(+)$

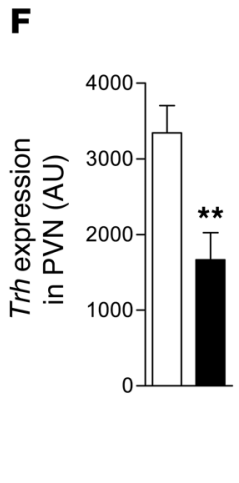

G

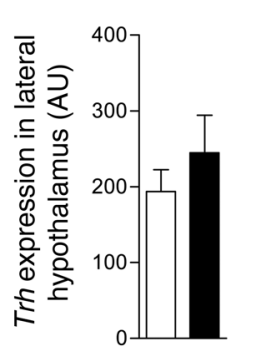

H

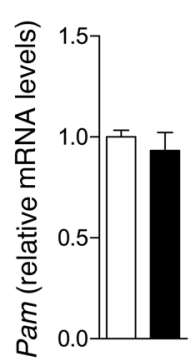

I

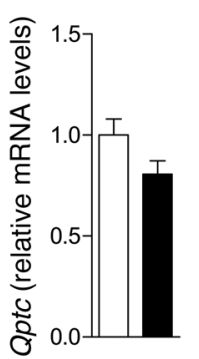

$\mathbf{J}$

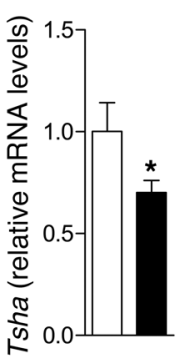

$\mathbf{K}$

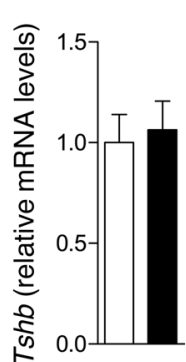

\section{Figure 3}

Suppression, stimulation, and mRNA levels of Tsh and Trh in pit-D2 KO mice versus CGA-cre controls. (A and B) Serum T4 and T3 levels after 4-hour administration of saline, T3, or T4 $(n=3-7)$. (C) Serum TSH levels before (B) and after (A) 4-hour administration of saline, T3, or T4 $(n=4-10)$. Mean \pm SEM serum TSH in the pit-D2 KO group after T4 treatment is indicated at right. (D) TSH serum levels before and 30 minutes after TRH stimulation $(n=4)$. (E) Representative in situ hybridization of Trh mRNA in the MBH. III, third ventricle. (F) Quantification of data in $\mathbf{E}$ $(n=7-9)$. (G) Quantification of Trh mRNA in the lateral hypothalamus $(n=7-9)$. (H and I) Relative expression of the TRH biosyntheses enzymes Pam and Qpct in the hypothalamus $(n=5)$. ( $\mathbf{J}$ and $\mathbf{K})$ Relative mRNA expression of Tsha and Tshb in the pituitary $(n=5-7)$. mRNA levels were normalized to CypA. ${ }^{*} P<0.05$, ${ }^{* *} P<0.01$ vs. control. 


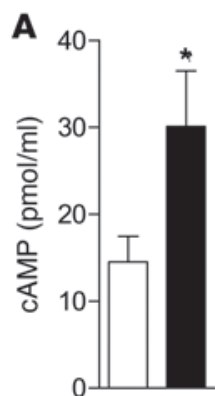

Control
B

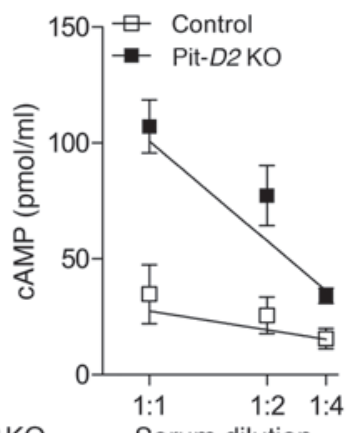

Pit-D2 KO

Serum dilution

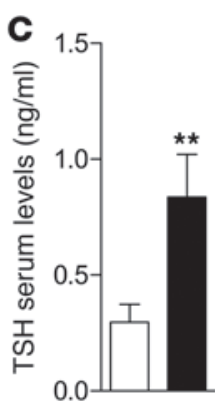

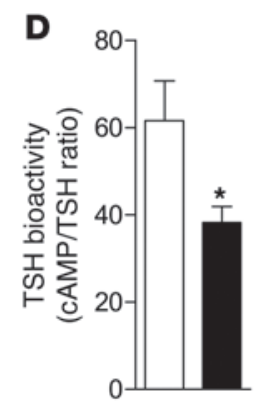

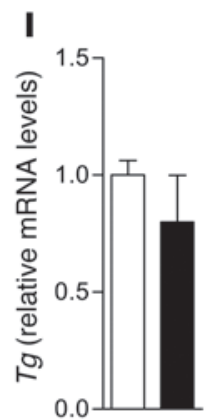

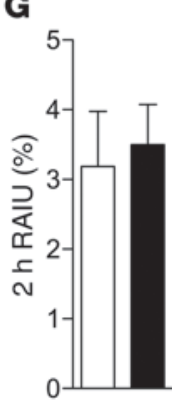

H

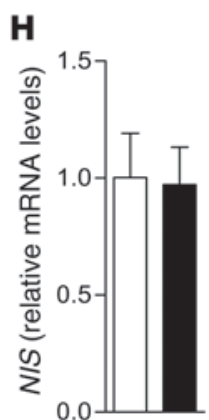

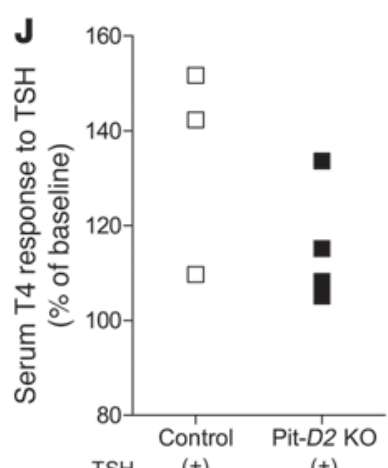

(+)
$\mathbf{E}$

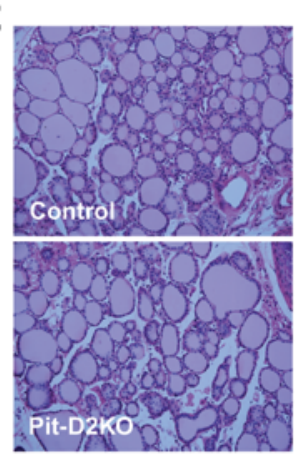

Figure 4

Thyroid parameters and response to TSH in pit-D2 KO mice versus CGA-cre controls. (A) cAMP levels, (B) serum dilution vs. cAMP production $(n=5)$, (C) TSH serum levels, and (D) TSH bioactivity $(n=6-8)$. (E) Representative structural analysis of thyroid glands, as assessed by optical microscopy $(n=3-5)$. H\&E staining (original magnification, $\times 20)$. (F) Relative thyroid weight $(n=8-10)$. (G) Thyroidal ${ }^{125}$ I uptake (RAIU) after 2 hours $(n=5)$. Uptake was calculated as the ratio of thyroid cpm to total cpm and expressed as a percentage. (H and I) mRNA expression of NIS and $T g(n=7-8)$, normalized to CypA. ( $\mathbf{J}$ and $\mathbf{K})$ T4 and T3 levels 2 hours after TSH administration. ${ }^{*} P<0.05$, ${ }^{* *} P<0.01$ vs. control.

T4-mediated negative feedback on PVN TRH expression. These mechanisms define a critical coordination between thyrotroph and tanycyte D2 pathways in the HPT and illustrate how preserving circulating serum T3 is indeed a centerpiece in thyroid hormone homeostasis.

The negative feedback mechanism in the thyrotroph is mediated by 2 important signals - serum T3 and serum T4 - via the D2 pathway (4). Thus, disruption of D2 in thyrotrophs would decrease their sensitivity to T4 and elevate serum TSH, which would in turn elevate serum T4 and T3 until a new steady state was reached at hyperthyroid levels. Alternatively, if serum T4 could bypass the thyrotroph and signal a negative feedback via the D2 pathway at the $\mathrm{MBH}$, this would compensate for the lack of thyrotroph D2 and preserve euthyroidism. Our newly developed pit-D2 KO mice demonstrated that the latter mechanism is the case: serum T3 remained normal in these animals, and they were systemically euthyroid (Figure 2, I and J, and Tables 1-3). That the pit-D2 KO mouse exhibits increased $\mathrm{T} 4$ signaling in the $\mathrm{MBH}$ was confirmed by the reduction in PVN Trh mRNA (Figure 3, E and F). The observation that serum $\mathrm{T} 4$ was elevated in the pit-D2 KO mouse (Figure $3 \mathrm{C}$ ) indicated that this $\mathrm{MBH}$-based compensatory mechanism is less sensitive to serum T4, a phenomenon that could be explained by MBH D3 activity inactivating $\mathrm{T} 4$ en route to the tanycytes.

However, if the MBH D2 pathway compensates for thyrotroph D2 pathway inactivation, then why were elevated serum TSH levels observed in pit- $D 2 \mathrm{KO}$ mice? The answer involves the posttranslational modification of the TSH molecule (i.e., TSH glycosylation), which is controlled by TRH (18). A reduction in TRH such as that seen during central hypothyroidism is expected to decrease TSH biological activity and prolong its half-life (18). In fact, pit-D2 KO TSH demonstrated approximately $40 \%$ decreased biological activity (Figure 4, A-D). This notion is strongly supported by the finding that most thyroidal parameters tested in the pit- $D 2 \mathrm{KO}$ mouse were normal, including thyroid structure, 2 -hour ${ }^{125}$ I uptake, and NIS and Tg mRNA levels (Figure 4, E and G-I), despite much higher serum TSH (Figure $2 \mathrm{~F}$ ). Thus, the new steady state reached by the pit-D2 KO mouse included normal pituitary-driven thyroidal activity, presumably because the elevation in serum TSH was mitigated by its reduced biological activity. Nevertheless, the pit- $D 2$ KO mice exhibited goiter, with an approximately $60 \%$ increase in thyroid size (Figure 4F). This raises several possibilities, including selective TSH activation of thyroidal growth versus activity resulting from the expected alteration in TSH glycosylation, or earlier thyroid growth (before the new steady state was achieved).

Despite the elevation in serum T4, pit-D2 KO mice exhibited strong evidence for systemic euthyroidism, as documented by normal serum T3, and tissue euthyroidism, given the multiple T3-responsive parameters that were normal (Figure 2, I and J, and Tables 1-3). This indicates that in response to a disruption in pituitary T4 signaling, multiple adjustments take place in the HPT, eventually preserving systemic euthyroidism. In fact, the pit-D2 KO mouse model represented yet another example that the HPT is wired to maintain normal serum T3 levels, in addition to most animal models of isolated or combined deiodinase deficiency (19). 


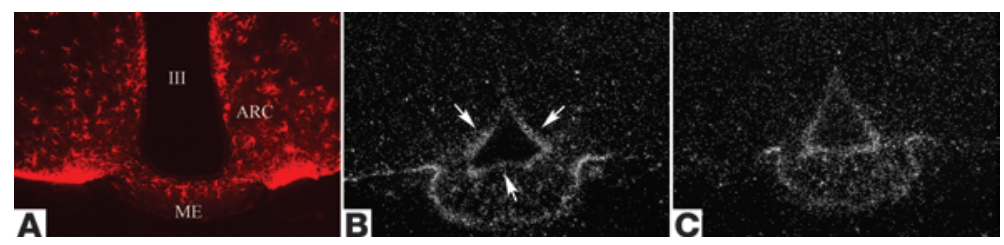

Figure 5

D2 and GFAP expression in the brain. (A) GFAP immunostaining demonstrated that in GFAP-cre animals, GFAP was expressed by astrocytes around the third ventricle - in the arcuate nucleus (ARC) and internal zone of the median eminence (ME) - but was not expressed by tanycytes located in the wall of the third ventricle. (B and $\mathbf{C}$ ) Darkfield image of radioactive in situ hybridization demonstrated that $D 2$ mRNA was expressed at the same level in the tanycytes of GFAP-cre control (B) and astro-D2 KO (C) mice. Arrows indicate D2 mRNA labeling in tanycyte cell bodies.

In the MBH, D2 has access to plasma-born or cerebral spinal fluidborn T4 to produce and release T3 that may then reach the PVN or the pituitary gland via portal blood (8). Previous studies in which MBH D2 activity is stimulated, either by LPS administration (20) or as a result of fatty acid amide hydrolase gene inactivation (21), indicate that D2-generated T3 can suppress TRH expression (22). In this region, D2 is reported to be expressed both in astrocytes (7), the only D2-expressing cell population that expressed GFAP-cre in the present studies (Figure 5A), and in tanycytes $(5,6)$. The preservation of $\mathrm{MBH} D 2$ activity in the astro- $D 2 \mathrm{KO}$ mouse (Figure $6 \mathrm{D}$ ) indicates that D2 is primarily expressed in tanycytes, although we cannot exclude with absolute certainty the possibility that selective ablation of D2 in hypothalamic astrocytes led to upregulation of D2 enzymatic activity in tanycytes to compensate for this loss. Nevertheless, by in situ hybridization histochemistry, no apparent increase in D2 mRNA was observed in astro-D2 KO versus GFAP-cre control tanycytes (Figure $5, \mathrm{~B}$ and $\mathrm{C}$ ). We are confident that astrocyte D2 in the hypothalamus is markedly reduced in the astro- $\mathrm{D} 2 \mathrm{KO}$ mouse, given that $\mathrm{D} 2$ activity was dramatically reduced in every other location in which it is expressed in glial cells (including the cerebral cortex, hippocampus, and cerebellum), but remained unaffected in the pituitary gland and outside the central nervous system (Figure 6, A-C, E, and F). At the same time, we cannot exclude the possibility that MBH D2-generated $\mathrm{T} 3$ also reaches the thyrotroph via portal blood and mediates, at least in part, the T4-mediated TSH negative feedback in pit- $D 2 \mathrm{KO}$ mice.

TSH has been linked to significant extrathyroidal effects, namely, lipolysis $(23,24)$ and control of bone mass (25-27). Interestingly, the pit-D2 KO animals exhibited decreased fat mass and normal bone mass (Table 2), which could potentially shed some light on the roles played by TSH in these processes. However, the reduced TSH biological activity in the pit-D2 $\mathrm{KO}$ mouse prevents us from reaching definitive conclusions. At least it is clear that the elevated serum TSH we detected in the pit-D2 KO animals was not associated with increased bone mass, as would be expected by some $(28,29)$. Lastly, also unexpected was the observation that astro- $D 2 \mathrm{KO}$ mice exhibited reduced BMD (Table 2). Although it is difficult to speculate on the underlying mechanisms (the global D2 KO has normal BMD; ref. 25), it is known that bone remodeling is under central control via the sympathetic nervous system $(30,31)$, a pathway that is affected by thyroid hormone and D2 expression $(32,33)$. Alternatively, D2 is expressed in bone and mouse osteoblasts (34) as well as in the chicken developing growth plate (35). Thus, given the GFAP expression in osteocytes and in cartilage chondrocytes (36), it is conceivable that selective D2 inactivation in these cells could have resulted in a phenotype that was not apparent in the global D2 KO mouse.

In conclusion, our characterization of the pit- $D 2 \mathrm{KO}$ mouse revealed coordination between the D2-generated $\mathrm{T} 3$ pathways in the pituitary gland and $\mathrm{MBH}$. In the absence of the pituitary D2 pathway, tanycyte $\mathrm{D} 2$ played a greater role within the $\mathrm{MBH}$, reducing TRH expression and bringing the HPT to a new steady state in which serum T3 levels and euthyroidism were preserved. Included in this mechanism was an elevation in serum TSH and a reduction in its biological activity, which combined had a neutral net effect on thyroidal activity. Characterization of the astro- $D 2 \mathrm{KO}$ mouse was instrumental in allowing us to define that tanycytic D2, not astrocytic D2, is a critical component of the feedback regulation mechanism of thyroid hormone on hypophysiotropic TRH.

\section{Methods}

Animals. To establish an in vivo mouse model for cell type-specific deletion of $D 2$, first we generated $D 2^{f}$ mice by inserting loxP sites $(37,38)$ into the $D 2$ gene. The resulting $D 2^{f}$ mice were then crossed with transgenic mice expressing Cre recombinase in a cell type-specific manner. The mouse D2 mRNA is unusually long $(\sim 6.5 \mathrm{~kb})$ and contains a 8,071 -bp-long single intron between codons 74 and 75 (39), making it risky to flox the whole exon 2 in view of the possible homologous recombination inside the floxed fragment. Therefore, the $5^{\prime}$ loxP site was placed into the $3^{\prime}$ region of the intron, while the $3^{\prime}$ loxP site was introduced to the $5^{\prime}$ region of the $3^{\prime}$ untranslated region (UTR) (Figure 1). According to this strategy, the loxPflanked region contained the portion of the D2 coding region located in exon 2 (from codon 75 to the end of the protein) including the active center of the D2 enzyme required for catalytic activity (40). The presence of the $3^{\prime}$ loxP site in the 3' UTR did not alter D2 activity (data not shown).

$D 2^{f l}$ mice were crossed with CGA-cre (B6;SJL-Tg[Cga-Cre]3Sac/J; Jackson Laboratories; ref. 41) and GFAP-Cre (FVB-Tg[GFAP-cre]25Mes/J; Jackson Laboratories; ref. 42) transgenic mice, in order to eliminate D2 activity in the anterior pituitary (pit-D2 KO mice) and in GFAP-expressing astrocytes (astro-D2 KO mice), respectively. In all experiments, Cre littermates were used as controls. Except when stated otherwise, all mice were male, between 9 and 12 weeks of age at the time of the studies. Hypothyroidism was induced in some animals, as indicated, by adding $1 \%$ sodium perchlorate and $0.1 \%$ metimazol in drinking water for 30 days.

Body composition. Animals were fasted overnight, and total skeleton area, LBM, and fat mass were measured by dual energy X-ray absorptiometry (DEXA; Lunar Pixi). For the procedure, mice were anesthetized with ketamine/xylazine $(200 \mathrm{mg} / \mathrm{kg}$ and $7-20 \mathrm{mg} / \mathrm{kg})$ before imaging, as described previously (43).

Indirect calorimetry. Mice were individually housed and acclimatized in a calorimeter for 2 days before studies. Measurements of gas exchange were performed subsequently over the next 2 days as described previously (43). These studies were performed in a comprehensive lab animal monitoring system (CLAMS; Columbus Instruments), a computer-controlled open circuit calorimetry system. Metabolic profiles were generated based on realtime data obtained in successive 14-minute cycles. Studies were performed 

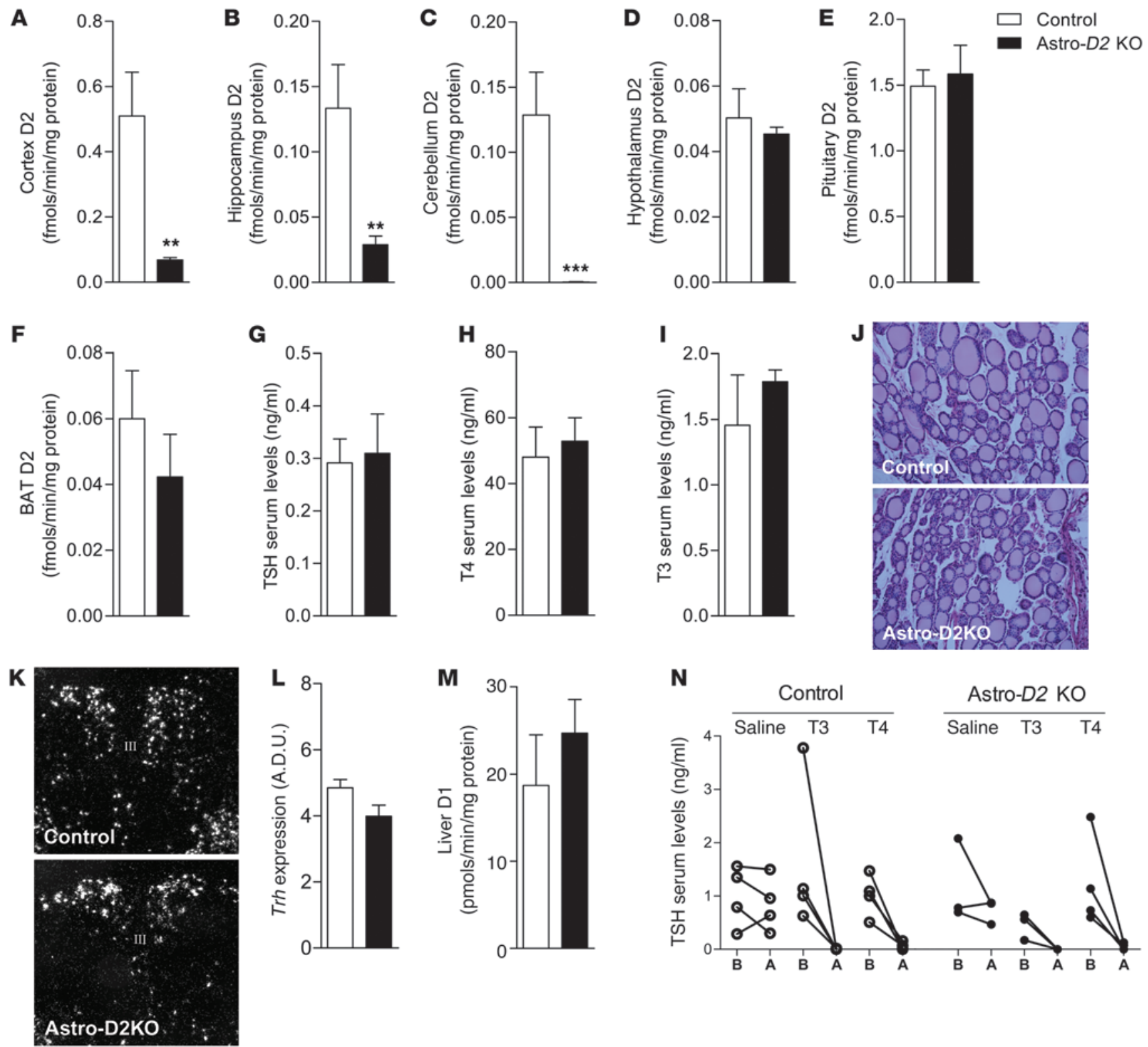

\section{Figure 6}

Thyroid phenotype in astro-D2 KO mice versus GFAP-cre controls. (A-F) D2 activity in sonicates of cortex, hippocampus, cerebellum, hypothalamus, pituitary, and BAT $(n=6)$. (G-I) Serum TSH, T4, and T3 levels $(n=6-7)$. (J) Representative structural analysis of thyroid glands, as assessed by optical microscopy $(n=3-5)$. H\&E staining (original magnification, $\times 20)$. (K) In situ hybridization of Thr mRNA in the PVN. Shown are coronal brain sections, including the MBH area and the third ventricle. (L) Computerized image analysis of the in situ hybridization autoradiograms ( $n=3-5$ per group). (M) Liver D1 activity $(n=6)$. (N) TSH serum levels before and after 4-hour administration of saline, T3, and T4 $(n=3-4) .{ }^{* \star} P<0.01,{ }^{* * *} P<0.001$ vs. control.

at $22^{\circ} \mathrm{C}$. The sensors were calibrated against a standard gas mixture containing defined quantities of $\mathrm{O}_{2}$ and $\mathrm{CO}_{2}$ (Airgas). $\mathrm{VO}_{2}$ was expressed as milliliters per kilogram per hour.

TSH suppression studies. TSH suppression studies were performed as previously described (11). Pit-D2 KO mice and CGA-cre controls, or astro-D2 KO mice and GFAP-cre controls, were placed on PTU (1 mg/liter drinking water) for 2 weeks. After blood was obtained from the orbital plexus $(\sim 150 \mu \mathrm{l})$, animals were injected s.c. with PTU $(1 \mathrm{mg} / 100 \mathrm{~g}$ BW; $0.1 \mathrm{ml}$ saline containing $0.01 \mathrm{~N} \mathrm{NaOH}) .1$ hour later, animals were injected s.c. with T3 $(1.2 \mu \mathrm{g} / 100 \mathrm{~g} \mathrm{BW}), \mathrm{T} 4$ (3 $\mu \mathrm{g} / 100 \mathrm{~g} \mathrm{BW})$, or vehicle. 4 hours later, new blood samples were collected, and animals were killed by $\mathrm{CO}_{2}$ inhalation.
TRH and TSH stimulation tests. TRH or TSH tests were performed as previously described (44). Control and pit-D2 KO mice received TRH $(5.0 \mu \mathrm{g} / \mathrm{kg}$ i.p.; Sigma-Aldrich) or bovine TSH $(250 \mathrm{mU} / 100 \mathrm{~g}$ i.p.; Sigma-Aldrich). Serum TSH, T4, and T3 levels were measured before and after TRH (30 minutes) or TSH (120 minutes) injection.

Thyroidal ${ }^{125}$ I uptake. Thyroidal ${ }^{125} \mathrm{I}$ uptake was performed as previously described (19) after injection of $\mathrm{Na}^{125} \mathrm{I}(15,000 \mathrm{cpm} / \mathrm{g}$ BW i.p.; NEN Life Science Products) into each animal. 2 hours later, mice were killed, and their thyroid glands were dissected and processed for radiometry. ${ }^{125}$ I uptake was calculated as the ratio of thyroid cpm to total cpm and expressed as a percentage. 
TSH biological activity. TSH biological activity was measured by bioassay using a line of $\mathrm{CHO}$ cells stably transfected with a human TSH receptor cDNA, as previously described (45). At the end of the incubation time, 220 $\mu \mathrm{l}$ of $10 \%$ trichloroacetic acid was added to each sample. cAMP was measured after adjusting to 6-7 $\mathrm{pH}$, using cAMP ${ }^{125}$ I RIA kit (PerkinElmer).

Charcoal uptake. The thyroxine binding protein was determined using a modification of previously described methods (46). $10 \mu \mathrm{l}$ mouse serum was diluted into $0.5 \mathrm{ml}$ of PBS ( $\mathrm{pH} 7.4$ ) containing approximately 7,000 cpm ${ }^{125} \mathrm{I}$ T4, with specific activity of $135-165 \mu \mathrm{Ci}(5.00-6.11 \mathrm{MBq})$ per microgram (Perkin Elmer). Samples were allowed to equilibrate for 45 minutes at room temperature and then transferred to an ice water bath for 15 minutes. Prechilled $0.025 \%$ activated charcoal $(0.5 \mathrm{ml}$; Sigma-Aldrich) solution in PBS was added, and samples were incubated on ice for an additional 15 minutes. Samples were spun in an Eppendorf centrifuge 5804R at $650 \mathrm{~g}$ for 15 minutes, and the charcoal-containing pellets were counted. Binding was calculated as the ratio of sample cpm to total cpm and expressed as a percentage.

Euthanasia and postmortem analyses. At the end of the experimental period, mice were euthanized by asphyxiation in $\mathrm{CO}_{2}$ chamber. Brain, liver, skeletal muscle, and epididymal adipose tissue was dissected, weighed, and frozen in liquid nitrogen, and organ weights were expressed relative to total BW. The thyroid gland was dissected, weighed, and fixed in $4 \%$ paraformaldehyde for 24 hours at $4^{\circ} \mathrm{C}$, and then embedded in paraffin, sectioned at $5 \mu \mathrm{m}$, and stained with $\mathrm{H} \& \mathrm{E}$. Blood was collected, and serum levels of TSH, T4, T3, FSH, and LH were measured using a MILLIPLEX rat thyroid hormone panel kit (Millipore Corp.), according to the manufacturer's instructions, and read on a BioPlex (BioRad).

In situ bybridization and analysis. In situ hybridization histochemistry was performed on every fourth section through the PVN or median eminence using a 741-base single-stranded [ ${ }^{35}$ S] UTP labeled cRNA probe for mouse TRH (47) or mouse D2 following previously described protocols (48). The coding region of the mouse D2 mRNA was amplified with Taq PCR (sense, ATGGGACTCCTCAGCGTAGACTT; antisense, GCTAATCTAGAATTCATCTCTTGC) and cloned into a pGEM-T vector with its $5^{\prime}$ end at the T7 promoter. The sequence was confirmed by sequencing. The construct was linearized with $\mathrm{NcoI}$ and subjected to transcription with SP6 polymerase to generate the antisense strand. In vitro transcription was performed using SP6/T7 systems (Promega Corp.) and $\left[{ }^{35} \mathrm{~S}\right]$ UTP $(1250 \mathrm{Ci} / \mathrm{mmol}$; PerkinElmer). The hybridization was performed under plastic coverslips in a buffer containing $50 \%$ formamide, a $2 \times$ concentration of standard sodium citrate, $10 \%$ dextran sulfate, $0.5 \%$ sodium dodecyl sulfate, $250 \mu \mathrm{g} / \mathrm{ml}$ denatured salmon sperm DNA, and $5 \times 10^{5}$ cpm radiolabeled probe for 16 hours at $56^{\circ} \mathrm{C}$. Slides were dipped into Kodak NTB autoradiography emulsion (Kodak) diluted 1:1 in distilled water, and the autoradiograms were developed after 3-7 days of exposure at $4^{\circ} \mathrm{C}$. Autoradiograms were visualized under darkfield illumination using a COHU 4910 video camera (COHU Inc.). Images were captured with a color PCI frame grabber board (Scion Corp.) and analyzed with a Macintosh G4 computer using Scion Image. Background density points were removed by thresholding the image and integrated density values (density $\times$ area) of hybridized neurons on each side of the PVN measured in 3 consecutive sections of the midportion of the PVN where hypophysiotropic TRH neurons have been identified in mice (47).

Immunofluorescence for GFAP. 25- $\mu \mathrm{m}$-thick coronal sections from the hypothalamus cut on a cryostat were collected in PBS and treated with $0.5 \%$ Triton-X-100 plus $0.5 \% \mathrm{H}_{2} \mathrm{O}_{2}$ in PBS for 15 minutes to increase antibody penetration. After rinses in PBS, sections were incubated in a mouse monoclonal antibody against GFAP (catalog no. MAB360; Millipore) overnight at a dilution of 1:2,000, diluted in $2 \%$ normal horse serum, $0.2 \%$ sodium azide, and $0.2 \%$ Photo-Flo. After rinses in PBS, sections were incubated in Cy3-conjugated donkey anti-mouse IgG (Jackson Immunoresearch) at 1:200 dilution for 2 hours. Sections were rinsed in PBS, mounted on glass slides, and coverslipped with Vectashield mounting medium (Vector).
Deiodinase assays. D1, D2, and D3 assays were performed in tissue sonicates in the presence of $10 \mathrm{mM}$ DTT and $0.25 \mathrm{M}$ sucrose as described previously (49). D1 and D2 assays were performed using $500 \mathrm{nM}{ }^{125} \mathrm{I}-\left(5^{\prime}\right)$ recombinant T3 (PerkinElmer) and $0.1 \mathrm{nM}{ }^{125} \mathrm{I}-\left(5^{\prime}\right) \mathrm{T} 4$ (PerkinElmer), respectively, as substrates and $1 \mathrm{mM}$ PTU for D2 measurements. D3 activity was measured as previously described (50) in the presence of $0.2 \mathrm{nM}$ ${ }^{125} \mathrm{I}-\left(5^{\prime}\right) \mathrm{T} 3$ (PerkinElmer) as substrate. Reactions were stopped by the addition of methanol, and the products of deiodination were quantified by UPLC (ACQUITY; Waters Corp.). Fractions were automatically processed through a Flow Scintillation Analyzer Radiomatic 610TR (PerkinElmer) for radiometry as described.

Gene expression analysis. Total RNA was extracted from pituitary and thyroid gland using RNAaqueous micro kit (Ambion; Life Technologies), according to the manufacturer's instructions. The extracted RNA was quantified with a NanoDrop spectrophotometer, and $1.0 \mu \mathrm{g}$ total RNA was reverse transcribed into cDNA using High Capacity cDNA reverse Transcription kit (Applied Biosystems). Genes of interest were measured by RT-PCR (Bio-Rad iCycler iQ Real-Time PCR Detection System) using iQ SYBR Green Supermix (Bio-Rad) with the following conditions: 15 minutes at $94^{\circ} \mathrm{C}$ (hot start), 30-50 seconds at $94^{\circ} \mathrm{C}, 30-50$ seconds at $55^{\circ} \mathrm{C}-60^{\circ} \mathrm{C}$, and $45-60$ seconds at $72^{\circ} \mathrm{C}$ for 40 cycles. A final extension at $72^{\circ} \mathrm{C}$ for 5 minutes was performed as well as the melting curve protocol to verify the specificity of the amplicon generation. Standard curves consisted of 4-5 points of serially diluted mixed experimental and control group cDNA. Cyclophilin A (CypA) was used as a housekeeping internal control gene. The coefficient of correlation was greater than 0.98 for all curves, and the amplication efficiency ranged between $80 \%$ and $110 \%$. Results were expressed as the ratio of test mRNA to CypA mRNA. mRNA levels of the following genes were measured: Tsha, Tshb, NIS, Tg, peptidylglycine alpha-amidating monooxygenase (Pam), and glutaminyl-peptide cyclotransferase (Qpct).

Statistics. All data were analyzed using PRISM software (GraphPad). Unless otherwise indicated, data represent mean \pm SEM. 1-way ANOVA followed by Student-Newman-Keuls test was used to compare differences among more than 2 groups; 2 -tailed Student's $t$ test was used to compare differences between 2 groups. A $P$ value less than 0.05 was considered significant.

Study approval. All experimental procedures were approved by the local IACUC (Miami, Florida, USA).

\section{Acknowledgments}

This work was supported by NIDDK grants R01 65055 and R01 37021, the National Science Foundation of Hungary (OTKA K81226), the Lendulet grant of Hungarian Academy of Sciences, and the European Community's Seventh Framework Programme (FP7/2007-2013) under grant agreement no. 259772. We are grateful to Judith Molina for skilled technical assistance and Susy Bianco for assistance with cAMP assay.

Received for publication October 24, 2012, and accepted in revised form January 31, 2013.

Address correspondence to: Antonio C. Bianco, Division of Endocrinology, Diabetes and Metabolism, University of Miami Miller School of Medicine, 1580 NW 10th Avenue, Suite 602, Miami, Florida 33136, USA. Phone: 305.243.5631; Fax: 305.243.7268; E-mail: abianco@deiodinase.org. Or to: Ronald M. Lechan, Division of Endocrinology, Diabetes and Metabolism, Tufts Medical Center, 800 Washington Street, Box 268, Boston, Massachusetts 02111, USA. Phone: 617.636.8517; Fax: 617.636.4719; E-mail: rlechan@tuftsmedicalcenter.org. 
1. Larsen PR. Thyroid-pituitary interaction: feedback regulation of thyrotropin secretion by thyroid hormones. NEngl J Med. 1982;306(1):23-32.

2. Geffner DL, Azukizawa M, Hershman JM. Propylthiouracil blocks extrathyroidal conversion of thyroxine to triiodothyronine and augments thyrotropin secretion in man. J Clin Invest. 1975;55(2):224-229.

3. Gereben B, et al. Cellular and molecular basis of deiodinase-regulated thyroid hormone signaling. Endocr Rev. 2008;29(7):898-938.

4. Christoffolete MA, et al. Atypical expression of type 2 iodothyronine deiodinase in thyrotrophs explains the thyroxine-mediated pituitary thyrotropin feedback mechanism. Endocrinology. 2006;147(4):1735-1743.

5. Guadano-Ferraz A, Obregon MJ, St Germain DL, Bernal J. The type 2 iodothyronine deiodinase is expressed primarily in glial cells in the neonatal rat brain. Proc Natl Acad Sci U S A. 1997; 94(19):10391-10396.

6. Tu HM, et al. Regional distribution of type 2 thyroxine deiodinase messenger ribonucleic acid in rat hypothalamus and pituitary and its regulation by thyroid hormone. Endocrinology. 1997; 138(8):3359-3368.

7. Diano S, Leonard JL, Meli R, Esposito E, Schiavo L. Hypothalamic type II iodothyronine deiodinase: a light and electron microscopic study. Brain Res. 2003;976(1):130-134

8. Fekete C, Lechan RM. Negative feedback regulation of hypophysiotropic thyrotropin-releasing hormone (TRH) synthesizing neurons: role of neuronal afferents and type 2 deiodinase. Front Neuroendocrinol. 2007;28(2-3):97-114.

9. Rodriguez EM, et al. Hypothalamic tanycytes: a key component of brain-endocrine interaction. Int Rev Cytol. 2005;247:89-164.

10. Kakucska I, Rand W, Lechan RM. Thyrotropinreleasing hormone gene expression in the hypothalamic paraventricular nucleus is dependent upon feedback regulation by both triiodothyronine and thyroxine. Endocrinology. 1992;130(5):2845-2850.

11. Schneider MJ, Fiering SN, Pallud SE, Parlow AF, St Germain DL, Galton VA. Targeted disruption of the type 2 selenodeiodinase gene (DIO2) results in a phenotype of pituitary resistance to T4. Mol Endocrinol. 2001;15(12):2137-2148.

12. Rosene ML, Wittmann G, Arrojo e Drigo R, Singru PS, Lechan RM, Bianco AC. Inhibition of the type 2 iodothyronine deiodinase underlies the elevated plasma TSH associated with amiodarone treatment. Endocrinology. 2010;151(12):5961-5970.

13. Bianco AC, Maia AL, da Silva WS, Christoffolete MA. Adaptive activation of thyroid hormone and energy expenditure. Biosci Rep. 2005;25(3-4):191-208.

14. Silva JE. Thermogenic mechanisms and their hormonal regulation. Physiological Review. 2006; 86(2):435-464.

15. Zavacki AM, et al. Type 1 iodothyronine deiodinase is a sensitive marker of peripheral thyroid status in the mouse. Endocrinology. 2005;146(3):1568-1575.

16. Shupnik MA, Chin WW, Habener JF, Ridgway EC. Transcriptional regulation of the thyrotropin subunit genes by thyroid hormone. J Biol Chem. 1985;260(5):2900-2903.

17. Shibusawa N, Yamada M, Hirato J, Monden T, Satoh T, Mori M. Requirement of thyrotropin- releasing hormone for the postnatal functions of pituitary thyrotrophs: ontogeny study of congenital tertiary hypothyroidism in mice. Mol Endocrinol. 2000;14(1):137-146.

18. Weintraub BD, Gesundheit N, Taylor T, Gyves PW. Effect of TRH on TSH glycosylation and biological action. Ann N Y Acad Sci. 1989;553:205-213.

19. Christoffolete MA, et al. Mice with impaired extrathyroidal thyroxine to 3,5,3'-triiodothyronine conversion maintain normal serum $3,5,3^{\prime}$-triiodothyronine concentrations. Endocrinology. 2007;148(3):954-960.

20. Fekete $C$, et al. Lipopolysaccharide induces type 2 iodothyronine deiodinase in the mediobasal hypothalamus: implications for the nonthyroidal illness syndrome. Endocrinology. 2004;145(4):1649-1655.

21. Sipe JC, Waalen J, Gerber A, Beutler E. Overweight and obesity associated with a missense polymorphism in fatty acid amide hydrolase (FAAH). Int J Obes (Lond). 2005;29(7):755-759.

22. Freitas BC, et al. Paracrine signaling by glial cellderived triiodothyronine activates neuronal gene expression in the rodent brain and human cells. J Clin Invest. 2010;120(6):2206-2217.

23. Iida Y, Amir SM, Ingbar SH. Stabilization, partial purification, and characterization of thyrotropin receptors in solubilized guinea pig fat cell membranes. Endocrinology. 1987;121(5):1627-1636.

24. Elgadi A, Zemack H, Marcus C, Norgren S. Tissuespecific knockout of TSHr in white adipose tissue increases adipocyte size and decreases TSHinduced lipolysis. Biochem Biophys Res Commun. 2010;393(3):526-530.

25. Bassett JH, et al. Optimal bone strength and mineralization requires the type 2 iodothyronine deiodinase in osteoblasts. Proc Natl Acad Sci U S A. 2010;107(16):7604-7609.

26. Bassett JH, et al. A lack of thyroid hormones rather than excess thyrotropin causes abnormal skeletal development in hypothyroidism. Mol Endocrinol. 2008;22(2):501-512.

27. Abe E, et al. TSH is a negative regulator of skeletal remodeling. Cell. 2003;115(2):151-162.

28. Sun L, et al. Intermittent recombinant TSH injections prevent ovariectomy-induced bone loss. Proc Natl Acad Sci U S A. 2008;105(11):4289-4294.

29. Sampath TK, et al. Thyroid-stimulating hormone restores bone volume, microarchitecture, and strength in aged ovariectomized rats. J Bone Miner Res. 2007;22(6):849-859.

30. Fonseca TL, et al. Double disruption of alpha2Aand alpha2C-adrenoceptors results in sympathetic hyperactivity and high-bone-mass phenotype. J Bone Miner Res. 2011;26(3):591-603.

31. Takeda S, et al. Leptin regulates bone formation via the sympathetic nervous system. Cell. 2002;111(3):305-317.

32. Silva JE. Thermogenesis and the sympathoadrenal system in thyrotoxicosis. In: Braverman LE, ed. Werner \& Ingbar's The Thyroid: A Fundamental And Clinical Text. Philadelphia, Pennsylvania, USA: Lippincott Williams and Wilkins; 2005:307-620.

33. Silva JE. Intermediary metabolism and the sympathoadrenal system in hypothyroidism. In: Braverman LE, ed. Werner \& Ingbar's The Thyroid: A Fundamental And Clinical Text. Philadelphia, Pennsylvania, USA: Lippincott Williams and Wilkins; 2005:817-823.

34. Gouveia $\mathrm{CH}$, et al. Type 2 iodothyronine seleno- deiodinase is expressed throughout the mouse skeleton and in the MC3T3-E1 mouse osteoblastic cell line during differentiation. Endocrinology. 2005;146(1):195-200.

35. Dentice $M$, et al. The Hedgehog-inducible ubiquitin ligase subunit WSB-1 modulates thyroid hormone activation and PTHrP secretion in the developing growth plate. Nat Cell Biol. 2005;7(7):698-705.

36. Kasantikul V, Shuangshoti S. Positivity to glial fibrillary acidic protein in bone, cartilage, and chordoma. J Surg Oncol. 1989;41(1):22-26.

37. Abremski K, Hoess R. Bacteriophage P1 sitespecific recombination. Purification and properties of the Cre recombinase protein. J Biol Chem. 1984;259(3):1509-1514.

38. Hoess RH, Abremski K. Mechanism of strand cleavage and exchange in the Cre-lox site-specific recombination system. J Mol Biol. 1985;181(3):351-362.

39. Davey JC, Schneider MJ, Becker KB, Galton VA. Cloning of a $5.8 \mathrm{~kb}$ cDNA for a mouse type 2 deiodinase. Endocrinology. 1999;140(2):1022-1025.

40. Croteau W, Davey JC, Galton VA, St Germain DL. Cloning of the mammalian type II iodothyronine deiodinase. A selenoprotein differentially expressed and regulated in human and rat brain and other tissues. J Clin Invest. 1996;98(2):405-417.

41. Cushman LJ, Burrows HL, Seasholtz AF, Lewandoski M, Muzyczka N, Camper SA. Cre-mediated recombination in the pituitary gland. Genesis. 2000;28(3-4):167-174.

42. Zhuo L, Theis M, Alvarez-Maya I, Brenner M, Willecke K, Messing A. hGFAP-cre transgenic mice for manipulation of glial and neuronal function in vivo. Genesis. 2001;31(2):85-94.

43. Castillo M, et al. Disruption of thyroid hormone activation in type 2 deiodinase knockout mice causes obesity with glucose intolerance and liver steatosis only at thermoneutrality. Diabetes. 2011;60(4):1082-1089.

44. Yamada M, et al. Tertiary hypothyroidism and hyperglycemia in mice with targeted disruption of the thyrotropin-releasing hormone gene. Proc Natl Acad Sci U S A. 1997;94(20):10862-10867.

45. Moeller LC, Kimura S, Kusakabe T, Liao XH, Van Sande J, Refetoff S. Hypothyroidism in thyroid transcription factor 1 haploinsufficiency is caused by reduced expression of the thyroidstimulating hormone receptor. Mol Endocrinol. 2003;17(11):2295-2302.

46. Jennings RD. A simple, rapid and precise automated method for the determination of T3-uptake. Ann Clin Biochem. 1977;14(5):263-268.

47. Kadar A, et al. Distribution of hypophysiotropic thyrotropin-releasing hormone (TRH)-synthesizing neurons in the hypothalamic paraventricular nucleus of the mouse. J Comp Neurol. 2010;518(19):3948-3961.

48. Fekete C, et al. Neuropeptide Y has a central inhibitory action on the hypothalamic-pituitary-thyroid axis. Endocrinology. 2001;142(6):2606-2613.

49. Curcio-Morelli C, et al. In vivo dimerization of types 1, 2, and 3 iodothyronine selenodeiodinases. Endocrinology. 2003;144(3):937-946.

50. Huang SA, et al. Transforming growth factorbeta promotes inactivation of extracellular thyroid hormones via transcriptional stimulation of type 3 iodothyronine deiodinase. Mol Endocrinol. 2005;19(12):3126-3136 